\title{
Effects of a New Topical Treatment Containing Several Hair Growth Promoters in Women with Early Female Pattern Hair Loss
}

\author{
Colombina Vincenzi ${ }^{a}$ Benedetta Marisaldi ${ }^{a} \quad$ Antonella Tosti $^{b}$ \\ Brijeshkumar Patel ${ }^{\mathrm{C}}$ \\ a Private Hospital Nigrisoli, Bologna, Italy; ${ }^{\mathrm{b}}$ Leonard M. Miller School of Medicine, University of Miami, Miami, FL, USA; \\ 'Private Consultant, Boca Raton, FL, USA
}

\section{Keywords}

Female pattern hair loss $\cdot$ Hair shedding visual scale $\cdot$ Hair mass index · Global photography · Hair growth promoters · Spectral.DNC-N ${ }^{\circledR}$

\section{Abstract}

Background: Female pattern hair loss (FPHL) is increasingly common and often presents with hair shedding. Spectral.DNC-N ${ }^{\circledR}$ contains anti-inflammatory actives and hair growth promoters. Objectives: To assess the efficacy and tolerability of Spectral DNC-N ${ }^{\circledR}$ (DS Laboratories, Inc.) in women with early FPHL and excessive shedding. Methods: Forty-nine patients were treated with Spectral DNC-N ${ }^{\circledR}$, applied twice daily for 3 months; 28 patients were included in the 3-month treatment extension. Efficacy assessments included the degree of hair shedding using the validated hair shedding visual scale, hair mass index (HMI), and photographic evaluation. Results: There was a statistically significant decrease in hair shedding and a corresponding increase in HMI by month 3, which was maintained through month 6. The mean investigator-assessed hair shedding score decreased from 3.5 to 2.0 and the hair mass increased from 75.8 to $84.3 \mathrm{~mm}^{2} / \mathrm{cm}^{2}$ by month 3 (both $p<0.01$ compared with baseline). By month 6 , the hair shedding score was reduced to 1.6 and the hair mass was maintained. Most patients (75\%) showed an increase in global hair density. Conclusions: Spectral.DNC-N ${ }^{\circledR}$ significantly reduced hair shedding, with a corresponding increase in hair mass and density. These effects were already evident after 3 months' treatment and further improved in those patients who continued treatment to month 6 . Tolerability was good and patient satisfaction levels were high.

c) 2018 S. Karger AG, Basel

\section{Introduction}

Female pattern hair loss (FPHL) is a common condition that causes considerable patient distress. It affects over 21 million women in the United States [1] and can often lead to a reduction in quality of life and secondary morbidity [2]. FPHL is significantly associated with metabolic syndrome and its medical complications $[3,4]$. It is characterized by slow progressive hair thinning that may or may not be associated with increased shedding, or by reduction in hair volume [5]. The prevalence of FPHL has been significantly increasing, suggesting that envi-

\section{KARGER}

(c) 2018 S. Karger AG, Basel

E-Mail karger@karger.com

www.karger.com/sad
Colombina Vincenzi

Ospedale Privato Nigrisoli

Viale Giambattista Ercolani 9

IT-40138 Bologna (Italy)

E-Mail colombina.vincenzi@ unibo.it 
ronmental factors may be involved [6]. It is slowly progressive and responds poorly to treatment. Hair loss pathophysiology involves many causal factors, and combination therapy targeting different pathways is likely to be optimal.

The only FDA-approved treatment for FPHL, topical minoxidil, is applied twice daily for a minimum period of 12 months. Although effective, women often do not tolerate topical minoxidil well, as it often causes itching and irritation. Studies indicate that minoxidil 5\% foam may be as effective, with a lower incidence of skin irritation and improved aesthetics [7-9]. Clinical effects may not be noticeable for up to 6 months of continued daily use. The clinical effects are also unpredictable, may be mildly unappreciated by the patient and, if treatment is not continued indefinitely, improvements will be lost. As a result, the majority of FPHL patients remain untreated.

Spectral.DNC-N ${ }^{\circledR}$ (DS Laboratories, Inc.) is a topical solution containing numerous hair growth promoters, including pyrrolidinyl diaminopyrimidine oxide, azelaic acid, lysophosphatidic acid, copper tripeptide-1, myristoyl pentapeptide-17, adenosine, piroctone olamine, retinol, and caffeine, in a novel nanosomal delivery system. These ingredients have been shown individually to stimulate hair growth and, in many cases, to improve the efficacy of minoxidil treatment when used in combination.

This study is the first to investigate the effects of Spectral.DNC- ${ }^{\circledR}$ in women with early FPHL who also complained of excessive shedding.

\section{Methods}

Female patients aged 18-60 years with early FPHL who complained of increased hair shedding were treated with Spectral. DNC-N ${ }^{\circledR}$ (1 mL twice daily applied to clean, dry hair) for 3 months, with the option to continue treatment for a further 3 months. FPHL was confirmed by trichoscopy and excessive shedding by a positive pull test. Patients with known hair shaft or scalp diseases other than FPHL and a history of minoxidil use (or other treatments affecting hair growth) within the last 6 months prior to study entry were excluded from the study. Patients could continue to use their normal shampoo and conditioners and dye their hair, maintaining the same color and hairstyle.

Primary efficacy assessments included investigator and patient assessment of degree of hair shedding based on a 10-point visual analog scale relating to the patient's hair type and length (short, medium, or long) [10], hair mass index (HMI) assessed by trichometry, and photographic evaluation. Patients were instructed to collect and bring to visit the hair shed during a shampoo before starting the study and before the 3- and 6-month follow-up visits. The hair was collected 2 days after previous shampoo. HMI is a direct measurement of hair mass and is determined by the number of hair fibers (density) and hair diameter. HMI is not influenced by the length of the hair. It was measured using a cross-section trichometer: a hand-held device "grabs" the bundle of hair from a $2 \times 2 \mathrm{~cm}$ scalp area in a J-slot and measures the cross-sectional area of the hair bundle. It then displays the trichometric index, which equals the bundle cross-sectional area in $\mathrm{mm}^{2} / \mathrm{cm}^{2}$ of scalp surface multiplied by 100 [11]. A separate locating strip allows repeated measurement of hair from the same scalp area without the use of tattoos.

The investigator-scored changes in hair density were evaluated from standardized photographs of a vertex view of the whole scalp, with hair parted in the center and combed away. Hair density was assessed using a 7 -point scale (where $-3=$ greatly decreased and $+3=$ greatly increased).

Secondary assessments included a patient questionnaire of overall satisfaction and specific product characteristics such as smell, texture, and ease of use. Patients were also asked whether treatment made their hair feel dirty or caused itching. Additional tolerability (dryness and erythema) and oiliness were assessed at months 3 and 6.

Data from investigator- and subject-assessed hair shedding scores, HMI, and average global hair density from baseline as well as 3 and 6 months following Spectral.DNC- $\mathrm{N}^{\circledR}$ treatment was statistically analyzed using two-way repeated-measures analysis of variance followed by Bonferroni post-test to compare means. A $p$ value $\leq 0.05$ was considered statistically significant. In case of secondary assessments, data were compared using the means and medians of perceptions scores provided by the subjects following the use of Spectral.DNC- ${ }^{\circledR}$.

\section{Results}

Forty-nine women with confirmed early FPHL and excessive shedding were enrolled in the 3-month study; 28 (57.1\%) patients elected to continue treatment for a further 3 months.

\section{Hair Shedding}

At baseline the mean scores for hair shedding as assessed by the investigator and patient were 3.5 and 3.8, respectively. After 3 months' treatment, a significant decrease in hair shedding score was noted. Mean scores were 2.0 and 2.1 , respectively ( $p<0.01$ versus baseline). There was further improvement in hair shedding in those patients who were treated for a total of 6 months with mean scores of 1.6 and $1.8(p<0.01)$ (Fig. 1$)$.

\section{Hair Mass Index}

As shown in Figure 2, there was a significant increase in HMI from baseline. HMI was increased from $75.83 \pm$ 25.10 to $84.31 \pm 23.83 \mathrm{~mm}^{2} / \mathrm{cm}^{2}$ at 3 months and further to $88.87 \pm 24.97 \mathrm{~mm}^{2} / \mathrm{cm}^{2}$ at the end of 6 months $(p<0.01$ versus baseline, $p<0.053$ months versus 6 months). 


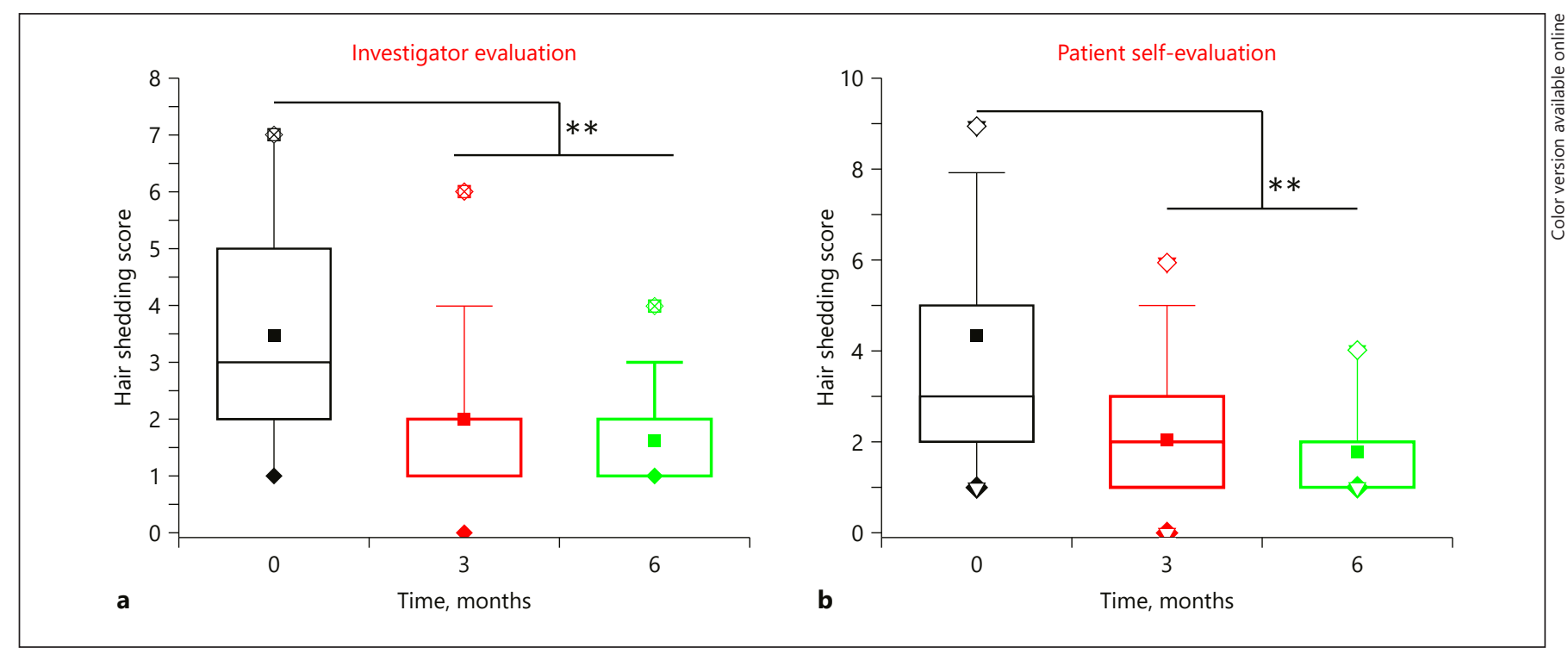

Fig. 1. Assessment of hair shedding by investigators (a) and patients (b) at baseline, month 3 , and month 6. Data represent means \pm standard deviations. ${ }^{* *} p<0.01$.

\section{Global Photographic Assessment}

As shown in Figure 3a, most patients (75\%) showed an increase in global hair density as evaluated by the investigator. At month 3,47\% of patients had moderately or greatly increased hair density. By month $6,96 \%$ of patients saw an improvement in their hair density. Also, the average hair density was significantly increased from baseline both at 3 and 6 months $(p<0.01)$. In addition, patients who continued treatment for 6 months showed statistically improved average global hair density in comparison to that observed at 3 months (Fig. 3b). A representative case study is shown in Figure 4, including videodermoscopy.

\section{Patient Satisfaction Assessment Questionnaire}

The mean satisfaction score at month 3 was 7.43. By month 6 , the mean score had increased to 8.12 (where $10=$ highest). More than $20 \%$ of patients rated their overall satisfaction as 10 (Figure 5).

The mean scores for each of the product characteristics assessed (i.e., smell, texture, and ease of use) were 7.4, 5.4, and 7.4, respectively (Figure 6). In addition, patients were asked whether the treatment made their hair dirty ( $3 / 49$ and $1 / 28$ patients at month 3 and 6 reported in the affirmative) or caused itching. The number of patients reporting no itching was $25 / 49$ (mean score 2.2) at month 3 and 18/28 (mean score 1.5) at month 6.

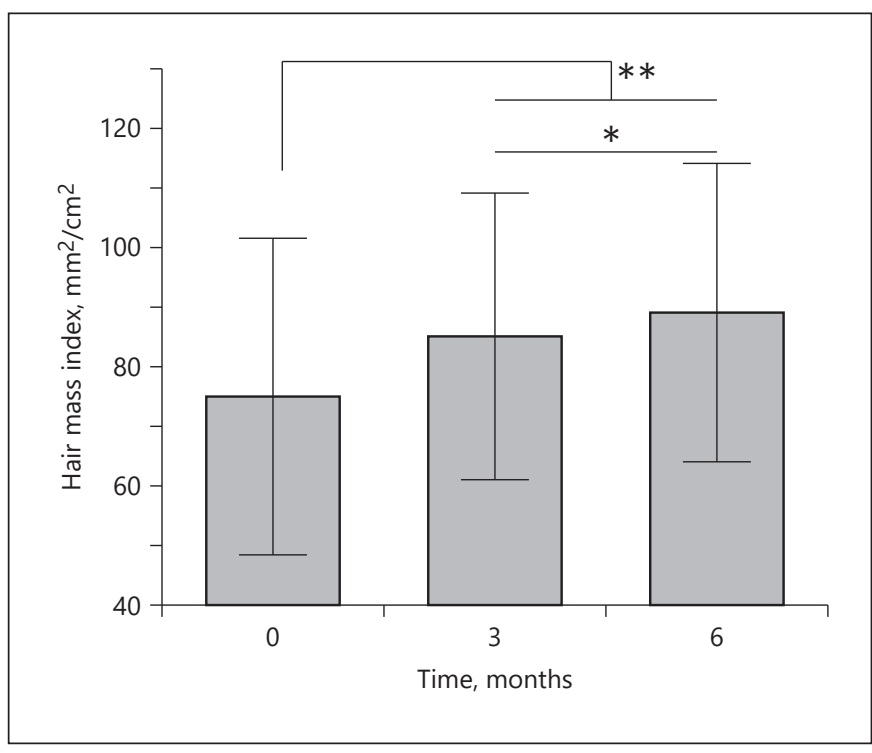

Fig. 2. Assessment of hair mass index at baseline, month 3 , and month 6. Data represent means \pm standard deviations. ${ }^{*} p<0.05$, ** $p<0.01$.

\section{Discussion}

FPHL is an increasing common and distressing condition that is difficult to treat successfully. The only approved treatment, topical minoxidil, has to be applied for 


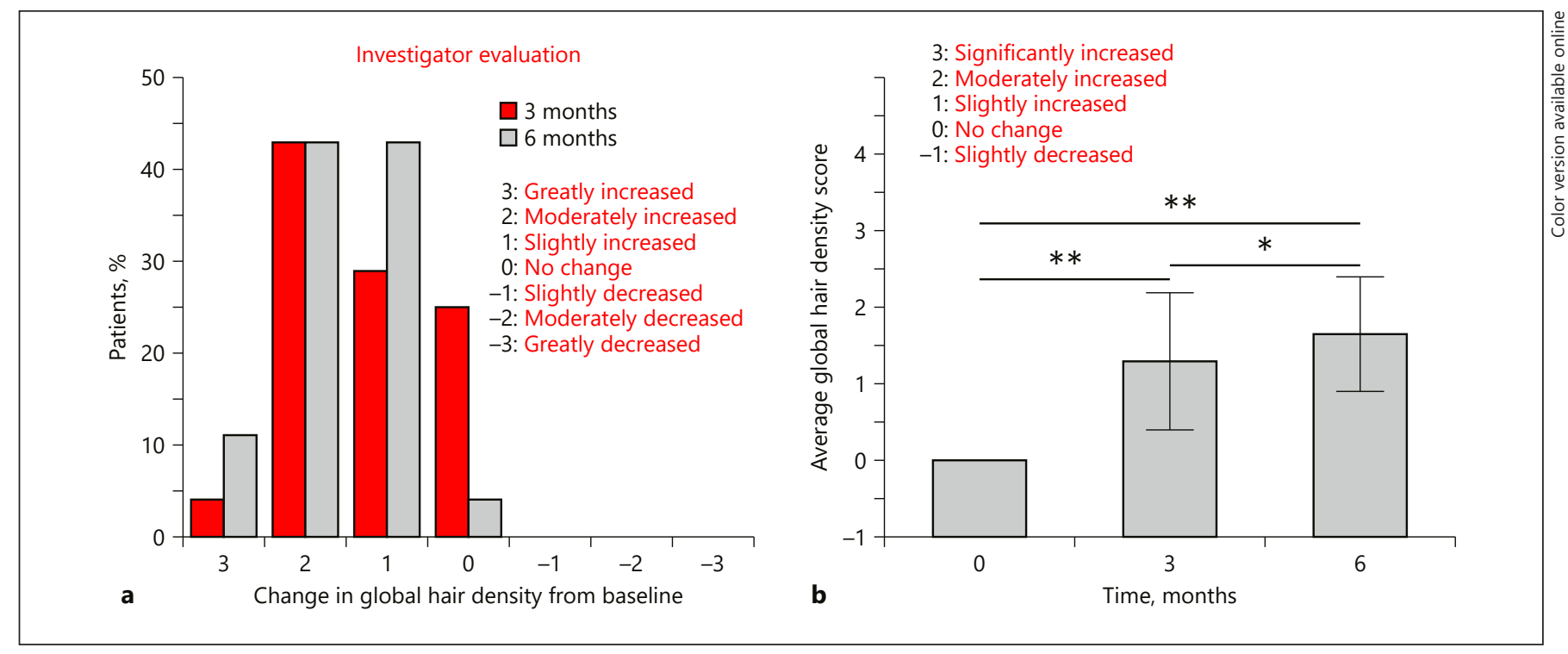

Fig. 3. Assessment of global hair density (a) and average hair density (b) at baseline, month 3 , and month 6 . Data represent means \pm standard deviations. ${ }^{*} p<0.05,{ }^{* *} p<0.01$.

Fig. 4. Photographs and videodermoscopy results of a representative case study at baseline, month 3, and month 6 .

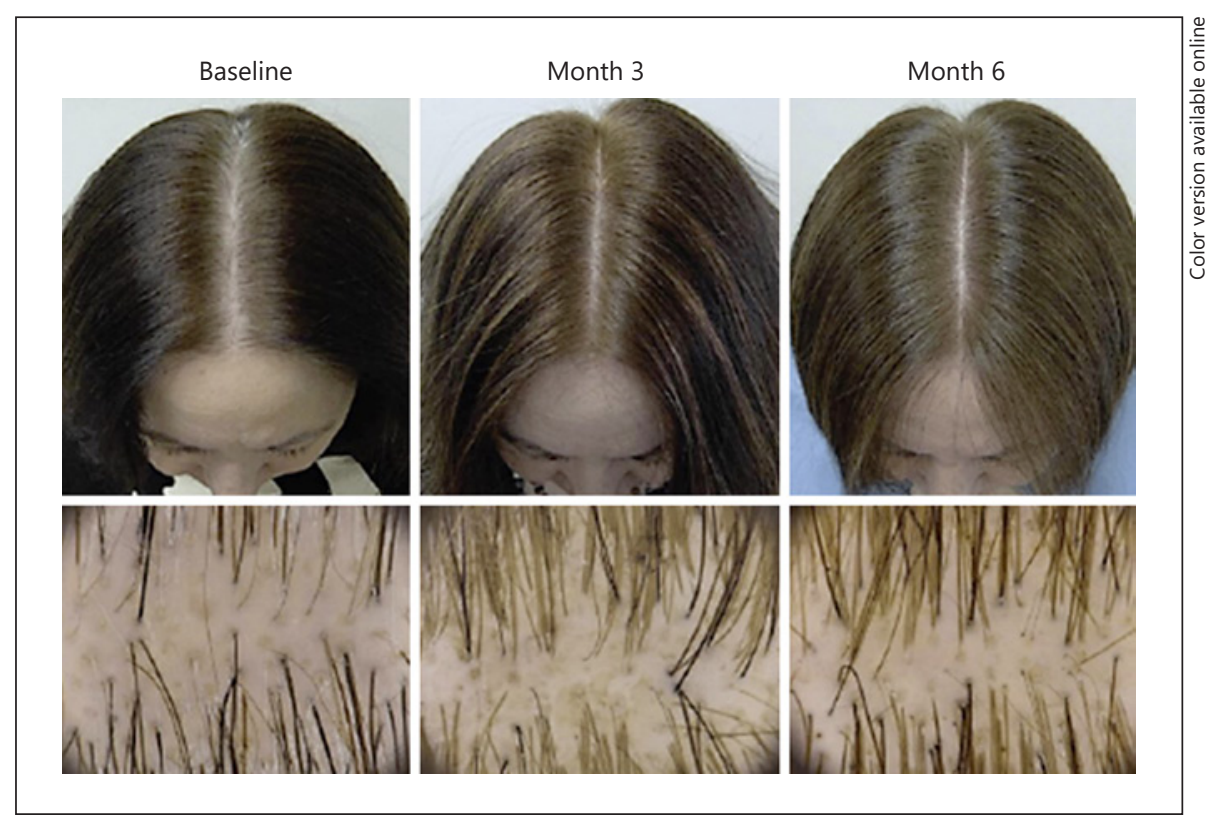

a minimum period of 12 months. Adherence can be problematic as it must be applied daily and it can take up to 6 months to see any benefit. If treatment is stopped, benefits can be lost.

Combination therapy is an important consideration as the causes of FPHL are multifactorial. Spectral.DNC$\mathrm{N}^{\circledR}$ contains 5\% nanoxidil (a liposome formulation containing a potassium channel opener, pyrrolidinyl diaminopyrimidine oxide, azelaic acid, lysophosphatidic acid, and copper tripeptide-1) in addition to a number of other recognized hair growth promoters and ingredients with anti-inflammatory properties. We were able to demonstrate a significant reduction in hair shedding and increased hair mass/density within 3 months $(p<0.01)$. In particular, the hair shedding score decreased by one-third from baseline by month 3 


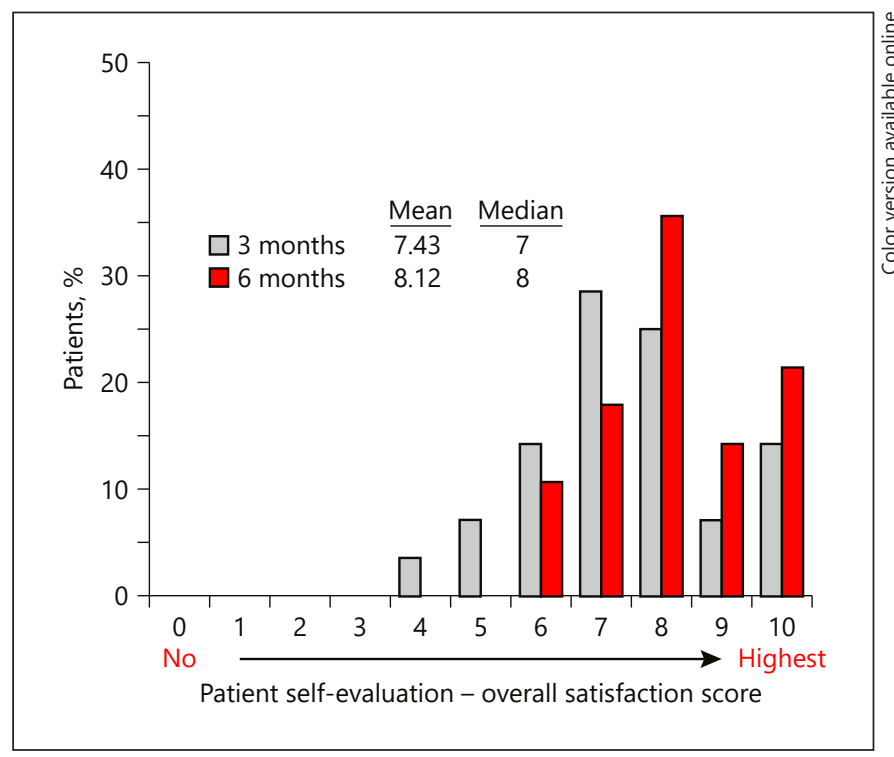

Fig. 5. Overall patient satisfaction scores at months 3 and $6(0=$ lowest, 10 = highest).

and by two-thirds by months 6 . The HMI increased by $>10 \%$ after 3 months, and this increase remained stable at 6 months. Hair density improved in almost half of the patients after 3 months and in nearly $100 \%$ of the patients after 6 months.

The regimen was well tolerated, and patients indicated a high degree of satisfaction. Investigator- and subjectassessed efficacy and subject satisfaction with this novel regimen provide clinicians with an effective treatment option for FPHL that also provides a high level of patient satisfaction, which may help promote patient adherence to long-term treatment.

Spectral.DNC-N ${ }^{\circledR}$ is a cosmetic treatment and we have no data so far to compare its efficacy to medical treatments such as topical minoxidil. The limitations of this study include that it was open and that the number of patients was small.

\section{Conclusion}

There is no cure for FPHL and patient expectations should be managed accordingly. The aim of treatment is to slow the rate of hair loss progression. Self-confidence may be improved by any resultant hair thickening. Three months' treatment with Spectral.DNC- $\mathrm{N}^{\circledR}$ considerably reduced hair shedding and increased hair mass and den-

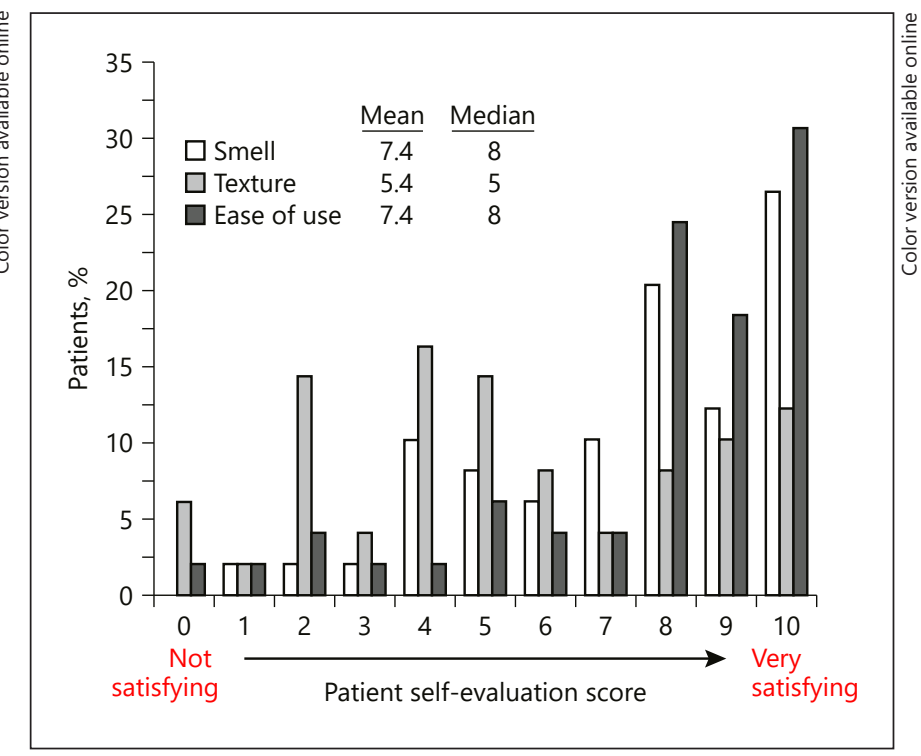

Fig. 6. Patient evaluation of specific product attributes at month 3 $(n=49 ; 0=$ not satisfied, $10=$ very satisfied $)$.

sity, with further improvements seen in patients who elected to continue treatment. Spectral.DNC-N ${ }^{\circledR}$ was well tolerated, with a high level of patient satisfaction.

\section{Acknowledgment}

This study was supported by a grant from DS Healthcare US.

\section{Statement of Ethics}

Patients provided written informed consent before study-related procedures were performed, and the protocol and consent were approved by the ethics committee at the investigation site. The study was conducted in accordance with the principles of good clinical practice and the Declaration of Helsinki.

\section{Disclosure Statement}

Dr. Tosti works as consultant for DS Laboratories, Inc. Dr. Patel was working as Senior Vice President, Research and Development, at DS Laboratories, Inc. at the time of study design/conception. Dr. Vincenzi and Dr. Marisaldi have no conflicts of interest to declare. 


\section{References}

1 Leavitt M: Understanding and management of female pattern alopecia. Facial Plast Surg 2008;24:414-427.

2 Hadshiew IM, Foitzik K, Arck PC, Paus R: Burden of hair loss: stress and the underestimated psychosocial impact of telogen effluvium and androgenetic alopecia. J Invest Dermatol 2004; 123:455-457.

3 Arias-Santiago S, Gutiérrez-Salmerón MT, Castellote-Caballero L, Buendía-Eisman A, Naranjo-Sintes R: Androgenetic alopecia and cardiovascular risk factors in men and women: a comparative study. J Am Acad Dermatol 2010;63:420-429.

4 Yi SM, Son SW, Lee KG, Kim SH, Lee SK, Cho ER, Kim IH, Shin C: Gender-specific association of androgenetic alopecia with metabolic syndrome in a middle-aged Korean population. Br J Dermatol 2012;167:306-313.
5 Blume-Peytavi U, Blumeyer A, Tosti A, Finner A, Marmol V, Trakatelli M, Reygagne $\mathrm{P}$, Messenger A: S1 guideline for diagnostic evaluation in androgenetic alopecia in men, women and adolescents. Br J Dermatol 2011; 164:5-15.

6 MacDonald A, Clark C, Holmes S: Frontal fibrosing alopecia: a review of 60 cases. J Am Acad Dermatol 2012;67:955-961.

7 Dinh QQ, Sinclair R: Female pattern hair loss: current treatment concepts. Clin Interv Aging 2007;2:189-199.

8 Lucky AW, Piacquadio DJ, Ditre CM, Dunlap F, Kantor I, Pandya AG, Savin RC, Tharp MD: A randomized, placebo-controlled trial of $5 \%$ and $2 \%$ topical minoxidil solutions in the treatment of female pattern hair loss. J Am Acad Dermatol 2004;50:541553.
9 Kanti V, Messenger A, Dobos G, Reygagne P, Finner A, Blumeyer A, Trakatelli M, Tosti A, Del Marmol V, Piraccini BM, Nast A, BlumePeytavi U: Evidence-based (S3) guideline for the treatment of androgenetic alopecia in women and in men - short version. J Eur Acad Dermatol Venereol 2018;32:11-22.

10 Martinez-Velasco MA, Vazquez-Herrera NE, Maddy AJ, Asz-Sigall D, Tosti A: The hair shedding visual scale: a quick tool to assess hair loss in women. Dermatol Ther 2017;7: 155-165.

11 Cohen B: The cross-section trichometer: a new device for measuring hair quantity, hair loss, and hair growth. Dermatol Surg 2008;34: 900-910. 